Mathematical Sciences And Applications E-Notes

VOLUMe 3 No. 2 PP. 54-57 (2015) (c) MSAEN

\title{
A NOTE ON LAGUERRE MATRIX POLYNOMIALS
}

\author{
ALİ ÇEVİK AND ABDULLAH ALTIN
}

(Communicated by İ. Onur KIYMAZ)

\begin{abstract}
In this paper, some new relations for Laguerre matrix polynomials are given.
\end{abstract}

\section{INTRODUCTION}

Recently, matrix polynomials that are solutions of a second order matrix differential equation are very popular subject in mathematics. In this area, many papers have been published ([17],[16],[18],[11],[19],[10],[23]). Many properties, extensions and generalizations of them have been introduced ([20],[9],[13],[12],,[22],[15],,[4],[24], $[2],[3],[1],[8])$. Laguerre matrix polynomial is one of them $([21],[20],[7],[5],[6])$.

In this paper, firstly a few lemmas are given. After, some new relations for Laguerre matrix polynomials are obtained by using these lemmas.

Throughout this paper, for a matrix $A \in \mathbb{C}^{r \times r}, \sigma(A)$ denotes the set of all eigenvalues of $A$ and is called its spectrum. $A$ is a positive stable matrix if $\operatorname{Re}(\lambda)>0$ for all $\lambda \in \sigma(A)$. Furthermore, the identity matrix and the null matrix in $\mathbb{C}^{r \times r}$ will be denoted $I$ and $\mathbf{0}$. If $A_{0}, A_{1}, \ldots, A_{n}$ are elements of $\mathbb{C}^{r \times r}$ and $A_{n} \neq \mathbf{0}$, then

$$
P(x)=A_{n} x^{n}+A_{n-1} x^{n-1}+\ldots+A_{1} x+A_{0}
$$

is called a matrix polynomial of degree $n$ in $x$ for every integer $n \geq 0$. From [17],

$$
(A)_{n}=A(A+I)(A+2 I) \ldots(A+(n-1) I) ; n \geq 1 ;(A)_{0}=I .
$$

is written. Using (1.1), we see that

$$
\frac{I}{(n-k) !}=(-1)^{k} \frac{(-n I)_{k}}{n !} ; 0 \leq k \leq n \text {. }
$$

In [17], if $f(z)$ and $g(z)$ are holomorphic functions which are defined in an open set $\Omega$ of the complex plane, and if $A$ is a matrix in $\mathbb{C}^{r \times r}$ for which $\sigma(A) \subset \Omega$, using the properties of the matrix functional calculus in [14] then

$$
f(A) g(A)=g(A) f(A) .
$$

Date: Received: April 17, 2015; Revised: May 1, 2015; Accepted: May 13, 2015.

2010 Mathematics Subject Classification. 33C45 (15A60, 15A09).

Key words and phrases. Laguerre matrix polynomials.

The authors are thankful for the referees for their helpful comments. 
Hence, if $B \in \mathbb{C}^{r \times r}$ is a matrix for which $\sigma(B) \subset \Omega$ and $A B=B A$, then

$$
f(A) g(B)=g(B) f(A) .
$$

Let $A$ be a matrix in $\mathbb{C}^{r \times r}$ satisfying $(-k) \notin \sigma(A)$ for every integer $k>0$ and $\lambda$ be a complex number whose real part is positive. In [17], $n$-th degree Laguerre matrix polynomial, $L_{n}^{(A, \lambda)}(x)$ is defined by

$$
L_{n}^{(A, \lambda)}(x)=\sum_{k=0}^{n} \frac{(-1)^{k}}{(n-k) ! k !}(A+I)_{n}(A+I)_{k}^{-1}(\lambda x)^{k} .
$$

By using (1.2), $L_{n}^{(A, \lambda)}(x)$ can be written in the form

$$
L_{n}^{(A, \lambda)}(x)=\frac{(A+I)_{n}}{n !} \sum_{k=0}^{n}(-n I)_{k}(A+I)_{k}^{-1} \frac{(\lambda x)^{k}}{k !} .
$$

Also, Laguerre matrix polynomials have the following derivative relation [20],

$$
\frac{d}{d x} L_{n}^{(A, \lambda)}(x)=-\lambda L_{n-1}^{(A+I, \lambda)}(x) \quad, \quad n \geq 1 .
$$

Lemma 1.1. [7] The raising operator for Laguerre matrix polynomials is

$$
\frac{d}{d x}\left(x^{A} e^{-\lambda x} L_{n}^{(A, \lambda)}(x)\right)=(n+1) x^{A-I} e^{-\lambda x} L_{n+1}^{(A-I, \lambda)}(x), \quad x>0
$$

where $A$ is positive stable matrix in $\mathbb{C}^{r \times r}$ and $\operatorname{Re}(\lambda)>0$.

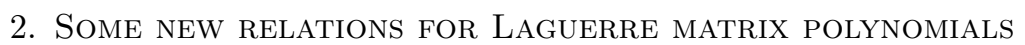

Lemma 2.1. Let $A$ be a matrix in $\mathbb{C}^{r \times r}$ satisfying the spectral condition

$$
\operatorname{Re}(\mu)>1 \text { for all } \mu \in \sigma(A) \text {, }
$$

and $\lambda$ be a complex number with $\operatorname{Re}(\lambda)>0$. For Laguerre matrix polynomials,

$$
\frac{d}{d x}\left[x^{A} L_{n}^{(A, \lambda)}(x)\right]=(A+n I) x^{A-I} L_{n}^{(A-I, \lambda)}(x) \quad, \quad x>0
$$

is satisfied.

Proof. We start by taking the derivative of $x^{A} L_{n}^{(A, \lambda)}(x)$ with respect to $x$. Thus, we have

$$
\begin{aligned}
\frac{d}{d x}\left[x^{A} L_{n}^{(A, \lambda)}(x)\right] & =\frac{d}{d x}\left[x^{A} \frac{(A+I)_{n}}{n !} \sum_{k=0}^{n}(-n I)_{k}(A+I)_{k}^{-1} \frac{(\lambda x)^{k}}{k !}\right] \\
& =\frac{d}{d x}\left[\frac{1}{n !} \sum_{k=0}^{n} \lambda^{k}(-n I)_{k}(A+I)_{n}(A+I)_{k}^{-1} \frac{x^{A+k I}}{k !}\right] \\
& =\frac{1}{n !} \sum_{k=0}^{n}\left\{\lambda^{k}(-n I)_{k}(A+I)_{n}(A+I)_{k}^{-1}(A+k I) \frac{x^{A+(k-1) I}}{k !}\right\}
\end{aligned}
$$


Then by using (1.1),

$$
\begin{aligned}
\frac{d}{d x}\left[x^{A} L_{n}^{(A, \lambda)}(x)\right] & =\frac{x^{A-I}}{n !} \sum_{k=0}^{n} \lambda^{k}(-n I)_{k}(A)_{n}(A+n I)(A)_{k}^{-1} \frac{x^{k}}{k !} \\
& =(A+n I) x^{A-I} \frac{(A)_{n}}{n !} \sum_{k=0}^{n}(-n I)_{k}(A)_{k}^{-1} \frac{(\lambda x)^{k}}{k !} \\
& =(A+n I) x^{A-I} L_{n}^{(A-I, \lambda)}(x) .
\end{aligned}
$$

holds. This completes the proof.

Theorem 2.1. Let $A$ be a matrix in $\mathbb{C}^{r \times r}$ satisfying the spectral condition (2.1) and $\operatorname{Re}(\lambda)>0$. Laguerre matrix polynomials satisfy the following relation with $x>0$

$$
A L_{n}^{(A, \lambda)}(x)=(A+n I) L_{n}^{(A-I, \lambda)}(x)+\lambda x L_{n-1}^{(A+I, \lambda)}(x), \quad n \geq 1 .
$$

Proof. The derivative of multiplication of $x^{A} L_{n}^{(A, \lambda)}(x)$ with respect to $x$ is, as follows from $(1.3)$,

$$
\begin{aligned}
\frac{d}{d x}\left[x^{A} L_{n}^{(A, \lambda)}(x)\right] & =A x^{A-I} L_{n}^{(A, \lambda)}(x)+x^{A} \frac{d}{d x} L_{n}^{(A, \lambda)}(x) \\
& =A x^{A-I} L_{n}^{(A, \lambda)}(x)-\lambda x^{A} L_{n-1}^{(A+I, \lambda)}(x), \quad n \geq 1 .
\end{aligned}
$$

Using (2.2) in the left side of this equation,

$$
A x^{A-I} L_{n}^{(A, \lambda)}(x)=(A+n I) x^{A-I} L_{n}^{(A-I, \lambda)}(x)+\lambda x^{A} L_{n-1}^{(A+I, \lambda)}(x), \quad n \geq 1
$$

is written. Then multiplying both sides with the inverse of $x^{A-I}$,

$$
A L_{n}^{(A, \lambda)}(x)=(A+n I) L_{n}^{(A-I, \lambda)}(x)+\lambda x L_{n-1}^{(A+I, \lambda)}(x), \quad n \geq 1
$$

is obtained.

Theorem 2.2. Let $A$ be a matrix in $\mathbb{C}^{r \times r}$ satisfying the spectral condition (2.1) and $\operatorname{Re}(\lambda)>0$. For Laguerre matrix polynomials,

$$
(n+1) L_{n+1}^{(A-I, \lambda)}(x)=(A+n I) L_{n}^{(A-I, \lambda)}(x)-\lambda x L_{n}^{(A, \lambda)}(x), \quad x>0
$$

holds.

Proof. Starting from the derivative of $e^{-\lambda x} x^{A} L_{n}^{(A, \lambda)}(x)$ with respect to $x$ and using Lemma 2.1, we can write

$$
\begin{aligned}
\frac{d}{d x}\left[e^{-\lambda x}\left(x^{A} L_{n}^{(A, \lambda)}(x)\right)\right] & =-\lambda e^{-\lambda x} x^{A} L_{n}^{(A, \lambda)}(x)+e^{-\lambda x} \frac{d}{d x}\left(x^{A} L_{n}^{(A, \lambda)}(x)\right) \\
(2.3) & =-\lambda e^{-\lambda x} x^{A} L_{n}^{(A, \lambda)}(x)+(A+n I) e^{-\lambda x} x^{A-I} L_{n}^{(A-I, \lambda)}(x) .
\end{aligned}
$$

Combining (1.4) and (2.3), then multiplying both side with $e^{\lambda x} x^{-A+I}$, the proof is completed. 


\section{REFERENCES}

[1] Aktaş, R., Çekim, B. and Çevik, A., Extended Jacobi matrix polynomials. Util. Math. 92 (2013), 47-64.

[2] Altın, A. and Çekim, B., Generating matrix functions for Chebyshev matrix polynomials of the second kind. Hacet. J. Math. Stat. 41 (2012), no. 1, 25-32.

[3] Altın, A. and Çekim, B., Some properties associated with Hermite matrix polynomials. Util. Math. 88 (2012), 171-181.

[4] Batahan, R.S., A new extension of Hermite matrix polynomials and its applications. Linear Algebra Appl. 419 (2006), 82-92.

[5] Çekim, B., New kinds of matrix polynomials. Miskolc Math. Notes 14 (2013), no. 3, 817-826.

[6] Çekim, B. and Altın, A., New matrix formulas for Laguerre matrix polynomials. Journal of Classical Analysis 3 (2013), no. 1, 59-67.

[7] Çekim, B., Altın, A. and Aktaş, R., Some relations satisfied by orthogonal matrix polynomials. Hacet. J. Math. Stat. 40 (2011), no. 2, 241-253.

[8] Çevik, A., Multivariable construction of extended Jacobi matrix polynomials. J. Inequal. Spec. Funct. 4 (2013), no. 3, 6-21.

[9] Defez, E. and Jódar, L., Some applications of the Hermite matrix polynomials series expansions. J. Comp. Appl. Math. 99 (1998), 105-117.

[10] Defez, E. and Jódar, L., Chebyshev matrix polynomials and second order matrix differential equations. Util. Math. 61 (2002), 107-123.

[11] Defez, E., Jódar, L. and Law, A., Jacobi matrix differential equation, polynomial solutions and their properties. Comput. Math. Appl. 48 (2004), 789-803.

[12] Defez, E., Jódar, L., Law, A. and Ponsoda, E., Three-term recurrences and matrix orthogonal polynomials. Util. Math. 57 (2000), 129-146.

[13] Defez, E., Hervás, A., Law, A., Villanueva-Oller, J. and Villanueva, R.J., Progressive transmission of images: PC-based computations, using orthogonal matrix polynomials. Mathl. Comput. Modelling 32 (2000), 1125-1140.

[14] Dunford, N. and Schwartz, J., Linear Operators. Vol. I, Interscience, New York, 1957.

[15] Grünbaum, F.A., Pacharoni, I. and Tirao, J.A., Matrix valued orthogonal polynomials of the Jacobi type. Indag. Math. (N.S.) 14 (2003), no. 3-4, 353-366.

[16] Jódar, L. and Company, R., Hermite matrix polynomials and second order matrix differential equations. J. Approx. Theory Appl. 12 (1996), no. 2, 20-30.

[17] Jódar, L., Company, R. and Navarro, E., Laguerre matrix polynomials and systems of second order differential equations. Appl. Num. Math. 15 (1994), 53-63.

[18] Jódar, L., Company, R. and Ponsoda, E., Orthogonal matrix polynomials and systems of second order differential equations. Differ. Equ. Dyn. Syst. 3 (1995), no.3, 269-288.

[19] Jódar, L. and Cortés, J.C., Closed form general solution of the hypergeometric matrix differential equation. Math. Comput. Modelling 32 (2000), 1017-1028.

[20] Jódar, L. and Defez, E., A connection between Laguerre's and Hermite's matrix polynomials. Appl. Math. Lett. 11 (1998), no. 1, 13-17.

[21] Jódar, L. and Sastre, J., On Laguerre matrix polynomials. Util. Math. 53 (1998), 37-48.

[22] Sayyed, K.A.M., Metwally, M.S. and Batahan, R.S., On generalized Hermite matrix polynomials. Electron. J. Linear Algebra 10 (2003), 272-279.

[23] Sayyed, K.A.M., Metwally, M.S. and Batahan, R.S., Gegenbauer matrix polynomials and second order matrix differential equations. Divulg. Mat. 12 (2004), 101-115.

[24] Taşdelen, F., Çekim, B. and Aktaş, R., On a multivariable extension of Jacobi matrix polynomials. Comput. Math. Appl. 61 (2011), no. 9, 2412-2423.

(A. ÇEVIK) Mersin University, Department of Mathematics, Çiftlikköy TR-33343 MERsin, TURKEY

E-mail address: cevik@mersin.edu.tr

(A. Altin) Ankara University, Faculty of Science, Department of Mathematics,

TANDOĞAN TR-06100 ANKARA, TURKEY

E-mail address: altin@science.ankara.edu.tr 